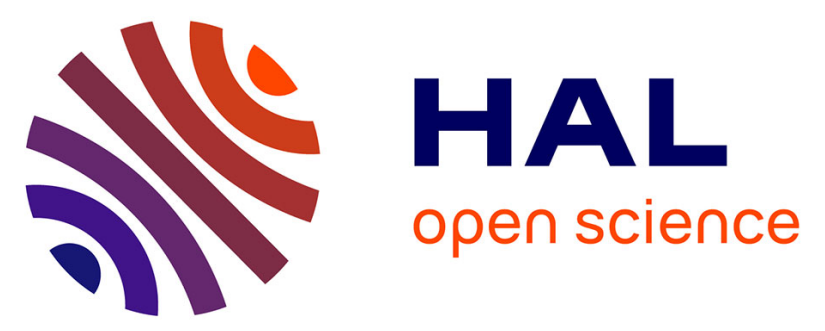

\title{
Toward the analysis of mitochondria isolated from leukemic cells with electrochemically instrumented microwell arrays
}

Gabriel Lemercier, Fadhila Sekli-Belaidi, Venkata S Vajrala, Emeline

Descamps, N. Sojic, Stéphane Arbault, Jean Emmanuel E Sarry, Pierre

Temple-Boyer, Jérôme Launay

\section{To cite this version:}

Gabriel Lemercier, Fadhila Sekli-Belaidi, Venkata S Vajrala, Emeline Descamps, N. Sojic, et al.. Toward the analysis of mitochondria isolated from leukemic cells with electrochemically instrumented microwell arrays. 19th International Conference on Solid-State Sensors, Actuators and Microsystems (Transducers 2017), Jun 2017, Kaohsiung, Taiwan. 4p., 10.1109/TRANSDUCERS.2017.7994374 . hal-01871405

\section{HAL Id: hal-01871405 \\ https://hal.laas.fr/hal-01871405}

Submitted on 10 Sep 2018

HAL is a multi-disciplinary open access archive for the deposit and dissemination of scientific research documents, whether they are published or not. The documents may come from teaching and research institutions in France or abroad, or from public or private research centers.
L'archive ouverte pluridisciplinaire HAL, est destinée au dépôt et à la diffusion de documents scientifiques de niveau recherche, publiés ou non, émanant des établissements d'enseignement et de recherche français ou étrangers, des laboratoires publics ou privés. 


\title{
TOWARD THE ANALYSIS OF MITOCHONDRIA ISOLATED FROM LEUKEMIC CELLS WITH ELECTROCHEMICALLY INSTRUMENTED MICROWELL ARRAYS
}

\author{
G. Lemercier ${ }^{1,2}$, F. Sekli-Belä̈di ${ }^{1}$, V.S. Vajrala ${ }^{3}$, E. Descamps ${ }^{1}$, N. Sojic ${ }^{3}$, S. Arbault ${ }^{3}$, J.E. Sarry ${ }^{2}$, \\ P. Temple-Boyer ${ }^{l}$, and J. Launay ${ }^{1, *}$ \\ ${ }^{1}$ LAAS-CNRS, Toulouse, FRANCE, \\ ${ }^{2}$ CRCT-INSERM, Toulouse, FRANCE and \\ ${ }^{3}$ ISM-CNRS, Bordeaux, FRANCE
}

\begin{abstract}
This work deals with the development of electrochemical transducers for the analysis of the metabolic status of mitochondria isolated from leukemic cells. It proposes the use of ring nanoelectrodes (RNE) integrated into microwell arrays for the simultaneous monitoring of the oxygen $\left(\mathrm{O}_{2}\right)$ consumption and the hydrogen peroxide $\left(\mathrm{H}_{2} \mathrm{O}_{2}\right)$ production. The sensor enabled the real-time recording of the oxygen consumption of approximately 10,000 isolated mitochondria. Solutions are now proposed to detect $\mathrm{H}_{2} \mathrm{O}_{2}$ production and to reduce the number of mitochondria under test, targeting the single mitochondrion analysis.
\end{abstract}

\section{KEYWORDS}

Bioelectrochemical nanosensor, microwell arrays, isolated mitochondria, acute myeloid leukemia

\section{INTRODUCTION \\ Motivation}

Recent studies introduced a direct relationship between the metabolic status of a sub-population of leukemic cells and its aptitude to overcome the chemotherapy [1]. Indeed, it has been shown that in acute myeloid leukemia (AML), the leukemic cells that exhibit the higher basal level of oxygen consumption rate (OCR) and mitochondrial respiratory capacity, are resistant to chemotherapy whereas glycolytic cells respond positively in vivo. These resistant leukemic cells are considered as the origin of the frequent relapses observed during treatment of AML and the cause of the patient death. Mitochondria are $1 \mu \mathrm{m}$ spherical organelles typically responsible of $90 \%$ of the oxygen consumption by the cell and directly involved in the mechanisms leading to production of $\mathrm{H}_{2} \mathrm{O}_{2}$, a member of the reactive oxygen species (ROS) family participating to cell signaling and oxidative stress [2]. The number of mitochondria contained in a single cell can vary from zero to a few thousand depending on its function. Innovative tools permitting to decipher cellular heterogeneity of cancer cells by single cell analysis are in constant and fast development [3] but mitochondrial heterogeneity also exists within a single cell [4,5]. Therefore, devices permitting the single mitochondrion analysis will offer the observation of biological processes at a new scale, to go further in the understanding of the mechanisms leading to chemoresistance in leukemia and cancer, but also of those involved in many diseases linked to mitochondrial dysfunction.

\section{Concept}

Electrochemistry provides in real time both quantitative and kinetic data. Moreover, the precise control of the applied potential provides the selectivity of species reacting at the working electrode surface. The concept presented here consists in the integration of ring nanoelectrodes into microwell array. Considering the location of mitochondria at the bottom surface of the microwell, the confinement related to the geometric structure maximize the collection ratio of the sensor since the diffusion layers almost totally close the microwell. In other words, almost all the species entering or exiting the microwell are detected by the RNE. The electrochemical microwells (ElecWell) are built on a transparent wafer in order to provide an optoelectrochemical system (Figure 1), firstly to be able to evaluate the fill rate of the microwells by mitochondria during an experiment but also to offer the possibility to simultaneously perform electrochemical and optical studies. Long-term experiments can be envisaged since the microscopic platform is temperature-controlled.

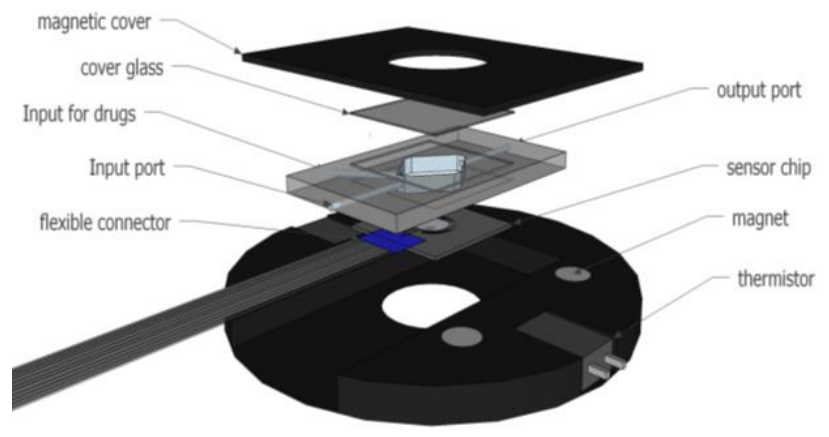

Figure 1: 3D drawing of the whole system comprising a temperature-controlled microscopic platform, the electrochemical sensor chip and a fluidic chamber

\section{EXPERIMENTAL}

Previous works [6] reported the design by multiphysics simulation, the manufacturing process and the electrochemical characterization of the first generation of the ElecWell. The following text will treat of the manufacturing of the second generation that is currently occurring.

\section{New chip design}

The chip dimensions are modified to be compatible with a commercial optofluidic system described thereafter. Nine square chips of $22 \mathrm{~mm} \mathrm{x} 22 \mathrm{~mm}$ 
presenting four different network configurations are manufactured within the same time from a four inches borosilicate wafer:

- 1 chip with 7 addressable microwells of $3 \mu \mathrm{m}$ diameter

- 2 chips with one network of 10,000 microwells of $6 \mu \mathrm{m}$ diameter

- $\quad 3$ chips with two networks of 10,000 microwells of $3 \mu \mathrm{m}$ diameter

- $\quad 3$ chips with 7 networks of 100 microwells of 3 $\mu \mathrm{m}$ diameter (Figure 2)

The center-to-center distance is now fixed at twentyfold the radius value (20R) to ensure hemispherical diffusion i.e. the absence of overlapping of the diffusion layers during electrolysis. Planar counter and pseudoreference electrodes (CE and RE respectively) are added to obtain an all-integrated three electrodes electrochemical setup.

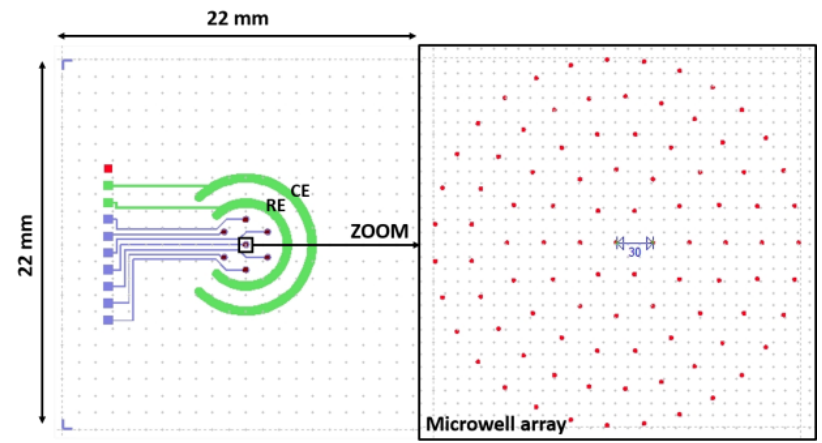

Figure 2: design of the chip containing seven arrays of 100 microwells with integrated $R N E$

\section{Device manufacturing}

Firstly, $370 \mathrm{~nm}$ of indium tin oxide (ITO), a transparent semiconductor, is deposited by means of reactive cathode sputtering on the entire wafer surface. Then, $2.5 \mu \mathrm{m}$ of silicon dioxide $\left(\mathrm{SiO}_{2}\right)$ is deposited by plasma enhanced chemical vapor deposition (PECVD) on the entire wafer surface. Then, a $200 \mathrm{~nm}$ layer of platinum (Pt) is deposited between two interfacial layers of $20 \mathrm{~nm}$ of titanium (Ti), used to ensure adhesion of platinum on $\mathrm{SiO}_{2}$. Physical vapor deposition and lift-off technique are used for such a purpose. Another $2.5 \mu \mathrm{m}$ of $\mathrm{SiO}_{2}$ is deposited on the entire surface by PECVD. Then, $20 \mathrm{~nm}$ of titanium and $120 \mathrm{~nm}$ of platinum are deposited following the previous described procedure to form planar counter and reference electrodes. A passivation layer of $100 \mathrm{~nm}$ of silicon nitride is deposited by inductivelycoupled plasma enhanced chemical vapor deposition (ICPECVD) and the active surface of the planar electrodes are defined by lift-off. Finally, a critical step of reactive ion etching (RIE) is performed to etch the stack $\mathrm{SiO}_{2} / \mathrm{Ti} / \mathrm{Pt} / \mathrm{Ti} / \mathrm{SiO}_{2}$ using mixture of gases as described previously [6]. This final RIE step results in the formation of the microwells but also in the definition of the RNE at mid-depth and the disc microelectrode (DME) made of ITO at the microwell bottom. The electrical contacts of the RNE are opened by wet etching using buffered oxide etchant.

After the cutting step of the chips and the connector assembly using silver paste, silver is electrochemically deposited onto platinum RE by chronoamperometry in aqueous solution containing $0.3 \mathrm{M}$ of silver nitrate $\left(\mathrm{AgNO}_{3}\right)$ previously deaerated. The $\mathrm{AgCl}$ layer is formed by linear voltammetry in $1 \mathrm{M} \mathrm{KCl}$ solution. Alternatively, the use of polypyrrole (Ppy) is considered to build a $\mathrm{Pt} / \mathrm{Ppy}$ quasi-reference electrode and avoid the dissolution of $\mathrm{AgCl}$ in solution during experiments [7].

\section{Electrodeposition of black platinum}

Black platinum (BIPt) is well known to enhance the catalytic activity of platinum regarding $\mathrm{H}_{2} \mathrm{O}_{2}$ and other ROS. The electrodeposition of platinum lead to the formation of a "cauliflower-like" platinum layer that enhances the surface/volume ratio. Electrodeposition at the RNE surface is carried out by chronoamperometry at $0.06 \mathrm{~V}$ vs $\mathrm{Ag} / \mathrm{AgCl}$ in aqueous solution containing 31 $\mathrm{mM}$ of hydrogen hexachloroplatinate and $1 \mathrm{mM}$ of lead acetate [8]. The process is automatically interrupted when the coulometric charge reaches $0.05 \mu \mathrm{C} / \mu^{2}{ }^{2}$. In that way the thickness of the layer deposited is precisely controlled and the too large shrinkage of the microwells opening is avoided.

\section{Functionalization of microwell bottoms}

To allow the rigorous normalization of the measurements, mitochondria have to be located only into the microwells. Several washings are performed to evacuate mitochondria located outside microwells, however those are also susceptible to provoke the escape of mitochondria from microwells. In order to avoid this possibility, antibodies directed against the mitochondrial voltage-dependent anion channel (VDAC), the most abundant protein of the mitochondrial outer membrane, are directly incorporated in electropolymerized polypyrrole (Ppy) on the ITO DME surface. A very thin layer is formed so that the bottom of the microwell remains transparent. To do this, the pyrrole monomer is diluted in aqueous solution at $20 \mathrm{mM}$ in presence of antiVDAC antibody at $25 \mu \mathrm{g} \cdot \mathrm{ml}^{-1}$. When the electropolymerization occurs, the antibody is believed to act as the counteranion so it is incorporated into the polymer matrix to ensure electroneutrality. The electropolymerization is carried out by chronoamperometry at $+2.0 \mathrm{~V}$ vs Pt for 250 milliseconds.

\section{Anti-biofouling layer at the inter-wells surface}

Mitochondria as well as many biological elements have the tendency to sediment onto surfaces and form a biofilm. During a typical experiment the normalization is permitted by optical observation through the rear face of the chip. If some mitochondria are located at the interwells surface, they won't be counted so the normalization and the measurement will be distorted. Thus, solutions are needed to render the chip surface non-adhesive to mitochondria while keeping the microwells inside intact. The chosen solution is the deposition of poly(L-lysine)graft-poly(ethylene-glycol) (PLL-g-PEG) by the technique of microcontact printing $(\mu \mathrm{CP})$. PEG is commonly used in order to produce anti-biofouling layers based on steric repulsion. To do so, polydimethylsiloxane 
(PDMS) is polymerized onto a flat silicon wafer previously treated with perfluorodecyltrichlorosilane (FDTS) to facilitate the demolding. Then, both the PDMS stamp and the sensor chip undergo a plasma $\mathrm{O}_{2}$ treatment to generate negative charges at their surface. The PDMS stamp is incubated for two minutes in PBS containing 1 $\mathrm{mg} \cdot \mathrm{ml}^{-1}$ of PLL-g-PEG. The stamp is then dried under a flux of nitrogen and the PLL-g-PEG layer is transferred onto the chip by applying a soft pressure for two minutes. Finally, the functionalization step is validated by the contact angle measurement of a water drop deposited onto the surface, which should be equal to approximately $35^{\circ}$.

\section{Fluidics}

Instead of using PDMS or other polymers such as SU8 or parylene to build a permanent fluidic on the chip, a commercial solution ensuring laminar flow was selected. This solution provided by Warner Instrument is originally designed for long-term studies of cell cultures by microscopy. Two microscopes slides on both sides of a RC-21B fluidic chamber are inserted in a temperaturecontrolled microscope platform. Within the context of our application the microscope slide constituting the bottom of the fluidic chamber is replaced by the sensor chip. Finally this solution ensure compatibility of the full system with a large range of microscopes and the nonpermanent characteristic of the fluidic make easy the reuse of the chips i.e. the cleaning and functionalization steps.

\section{RESULTS}

\section{Measurements of mitochondrial activity}

Mitochondria isolated from saccharomyces cerevisiae were used in this development phase for practical reasons. The OCR is measured by cyclic voltammetry at the RNE. Voltammetric scans are performed at $4 \mathrm{~V} / \mathrm{s}$ in the interval $[-1 ;-0.1] \mathrm{V}$ with a delay of 20s between scans in order to avoid the complete consumption of the oxygen within the microwells by the RNEs. The current value corresponding to the oxygen concentration in solution is taken at $-0.7 \mathrm{~V}$ vs $\mathrm{Ag} / \mathrm{AgCl}$ at each cycle.

Measurements of OCR by cyclic voltammetry at the RNE were obtained with 100x100 microwell networks (Figure 3). We notice that mitochondrial respiration starts after the addition of ethanol (1\%), a twofold increase is observed after addition of $1 \mathrm{mM}$ of adenosine diphosphate (ADP), then the respiration is inhibited after addition of $1.8 \mu \mathrm{M}$ of antimycin A. These results validated the ability of the system to analyze the metabolic features of mitochondria but they also evidenced the need to develop solutions to enhance the filling rate of the microwells and to avoid the sedimentation of interfering mitochondria at the inter-wells surface, what the next generation of the device should offer.

\section{Electrodeposition of black platinum}

The BlPt electrodeposited is characterized by scanning electron microscopy and electrochemistry in sulfuric acid, ferrocenedimethanol and $\mathrm{H}_{2} \mathrm{O}_{2}$ solutions. The ratio of the faradic current measured at $+0.35 \mathrm{~V}$ vs
$\mathrm{Ag} / \mathrm{AgCl}$ in $1 \mathrm{mM} \mathrm{H}_{2} \mathrm{O}_{2}$ solution before and after electrodeposition of BlPt is equal to two. This value can be increased by optimization of the composition of the solution used for electrodeposition but also by changing the coulometric charge deposited.
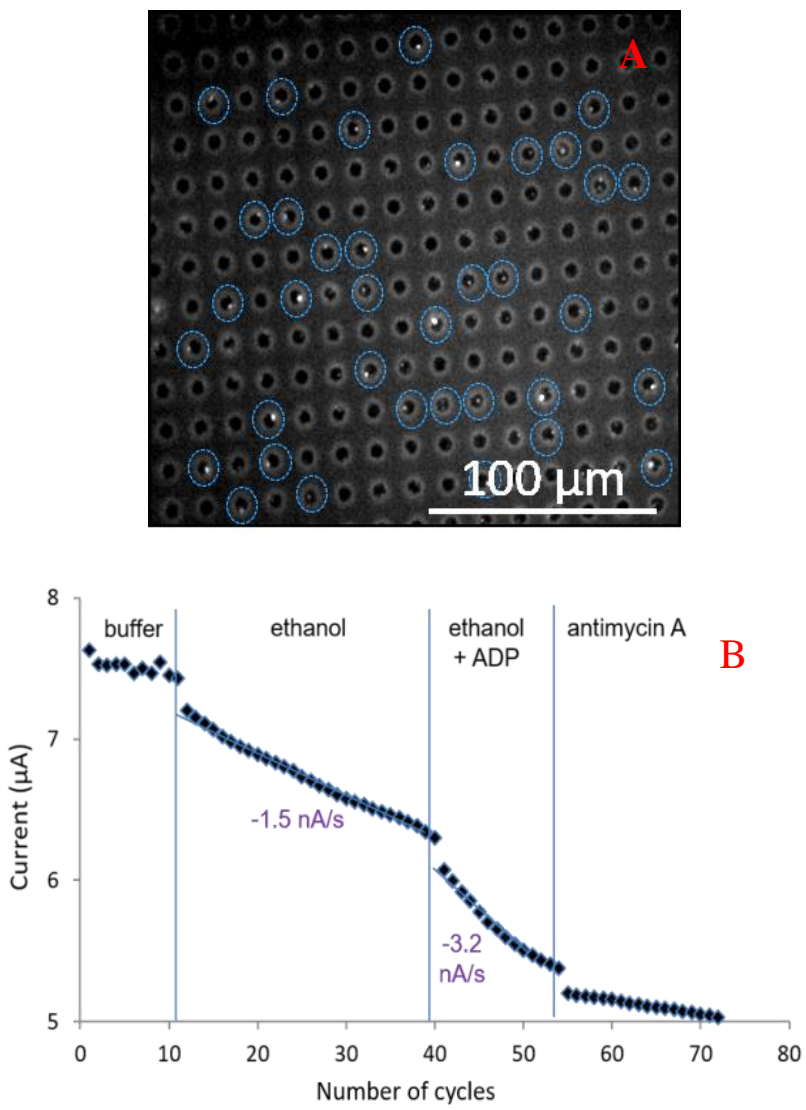

Figure 3: Simultaneous fluorescence microscopy (rear view through the chip) of mitochondria in microwells $(A)$ and OCR measurement by cyclic voltammetry at the RNE (B). Blue circles on A indicate the microwells filled by one mitochondrion.

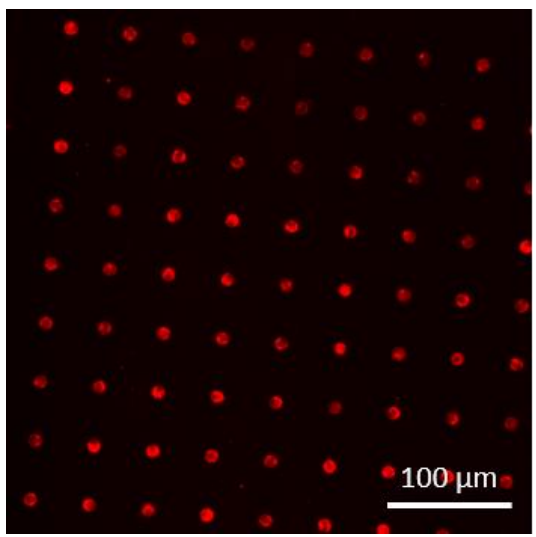

Figure 4: Fluorescence microscopy (front view) of antiVDAC antibodies immobilized into polypyrrole at the DME surface

Functionalization of microwell bottoms 
Anti-VDAC antibodies were successfully immobilized at the Pt DME surface (Figure 4) and ITO slides (result not shown) by following the procedure previously described. A secondary fluorescent antibody was used to check the presence and the accessibility of the primary one. A control experiment (result not shown) has been performed to verify the absence of non-specific interactions between the secondary antibody and Ppy electropolymerized in absence of the primary antibody. No non-specific interactions were detected, provided a preincubation with bovine serum albumin (BSA).

\section{Anti-biofouling layer at the inter-wells surface}

The efficacy of the anti-biofouling layer deposited by $\mu \mathrm{CP}$ was evaluated by optical observations conducted with isolated mitochondria. Firstly, $100 \mu \mathrm{l}$ of mitochondrial solution is deposited on the chip. Nothing is done for 3 minutes to allow mitochondrial sedimentation. Then, the solution is withdrawn by pipetting and a first observation is done to automatically count the number of particles stuck on top of the chip for a given surface $\left(18.75 \mathrm{~mm}^{2}\right)$. After that, the chip is washed thoroughly with PBS and mitochondrial particles are counted a second time. The figure 5 shows the results obtained when bare $\mathrm{SiO}_{2}$ is compared to a PLL-g-PEG modified one.

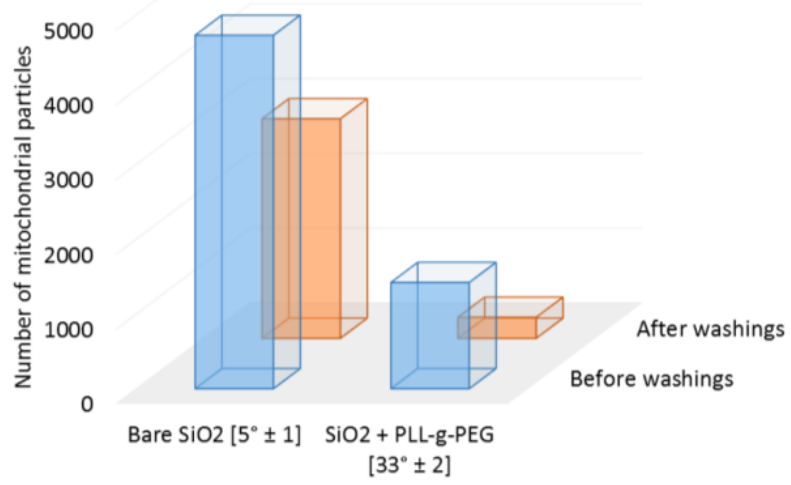

Figure 5: Number of mitochondrial particles counted before and after washings, with and without presence of a PLL-g-PEG layer. Static contact angle measurements are also displayed.

\section{DISCUSSION}

The first generation of the ElecWell permitted to validate the approach experimentally with the measurement of the OCR of approximately 10,000 isolated mitochondria in response to the additions of metabolic substrates and antimycin A. Nevertheless, interfering mitochondria prevented the rigorous normalization and the production of hydrogen peroxide has not been detected.

The second generation of the ElecWell device is expected to improve the manipulation of biological elements thanks to the addition of the microscope platform and the fluidic. The fill rate and the sensor sensitivity should also be enhanced thanks to the presence of anti-VDAC antibodies at the microwell bottoms and the BlPt at the RNE surface (Figure 6). Finally, the anti- biofouling layer should allow rigorous normalization of the measurements making the data obtained useful for biologists. All those developments merged herein allow us to believe in the final goal consisting in the analysis of mitochondria isolated from leukemic cells, at the single mitochondrion level.

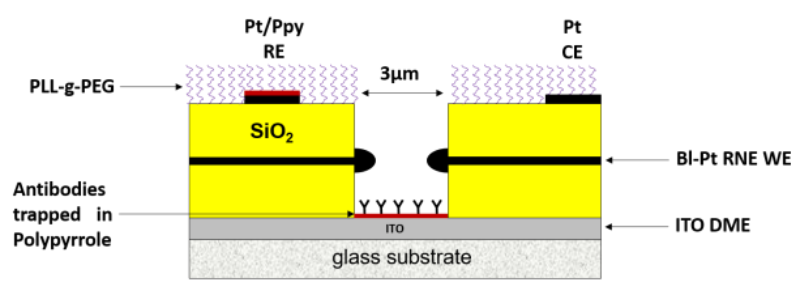

Figure 6: Schematic-sectional view of the second generation of the opto-electrochemical ElecWell device

\section{ACKNOWLEDGEMENTS}

This work was partly supported by LAAS-CNRS micro and nanotechnologies platform member of the French RENATECH network. We also want to thank the ONCODEVICE consortium.

\section{REFERENCES}

[1] Farge T. et al. "Chemotherapy resistant human acute leukemia cells are not enriched for leukemic stem cells but require oxidative metabolism", Cancer Discovery, Accepted.

[2] M. P. Murphy, "How mitochondria produce reactive oxygen species", Biochemical Journal, 2009.

[3] Fritzsch et al. "Single-cell analysis in biotechnology, systems biology, and biocatalysis", Annual review of chemical and biomolecular engineering, 2012.

[4] A. V. Kuznetsov and R. Margreiter, "Heterogeneity of mitochondria and mitochondrial function within cells as another level of mitochondrial complexity" International journal of molecular sciences, 2009.

[5] K. M. Fuller and E. A. Arriaga, "Advances in the analysis of single mitochondria" Current opinion in biotechnology, 2003.

[6] F. Sekli-Belaïdi et al. "Integration of ring nanoelectrodes into microwells for the bioelectrochemical analysis in sub-picolitre volumes", Sensors and Actuators B: Chemical, 2016.

[7] J. Ghilane et al. "Metal/polypyrrole quasi-reference electrode for voltammetry in nonaqueous and aqueous solutions" Analytical chemistry, 2006.

[8] S. Ben-Amor et al. « Enhanced detection of hydrogen peroxide with platinized microelectrode arrays for analyses of mitochondria activities" Electrochimica Acta, 2014.

\section{CONTACT}

*J. Launay, tel: +33-561336809; jlaunay@laas.fr 